REVIEW

\title{
Emergency thoracotomy: "how to do it"
}

D Wise, G Davies, T Coats, D Lockey, J Hyde, A Good

Emerg Med J 2005;22:22-24. doi: 10.1136/emj.2003.012963

This paper describes a simple approach to emergency thoracotomy that can be used by a doctor in the prehospital environment and in the resuscitation room.

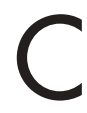
ardiac arrest after penetrating chest trauma may be an indication for emergency thoracotomy. A successful outcome is possible if the patient has a cardiac tamponade and the definitive intervention is performed within 10 minutes of loss of cardiac output. ${ }^{1-3}$

Wherever possible a patient needing surgery for penetrating chest trauma should be moved to an operating theatre where optimal surgical expertise and facilities are available. However, this is not an option if the patient is in cardiac arrest. Specialist cardiothoracic surgical help is not immediately available in most emergency departments, so the task of performing an emergency thoracotomy will fall to other specialists. The technique should therefore be as straightforward as possible.

The authors describe a simple approach to emergency thoracotomy that was originally developed for a doctor working single handed in the prehospital environment, but has been found to be equally useful in the resuscitation room. The technique has been used successfully in our practice for many years.

\section{EMERGENCY "CLAM SHELL" THORACOTOMY \\ Indication}

Penetrating chest/epigastric trauma associated with cardiac arrest (any rhythm).

\section{Contraindications}

- Definite loss of cardiac output for greater than 10 minutes.

- Any patient who has a cardiac output, including hypotensive patients.

- Blunt trauma.

See end of article for authors' affiliations

.....................

Correspondence to: Dr D Wise, Department of Emergency Medicine and Pre-hospital Care, Royal London Hospital, London E1 1BB, UK; david.wise@ bartsandthelondon.nhs.uk

Accepted for publication 22 March 2004
(2) Time should not be wasted on full asepsis (that is, fully preparing the skin and surgically draping the patient) but a rapid application of skin preparation is appropriate.

(3) Using a scalpel and blunt forceps make bilateral $4 \mathrm{~cm}$ thoracostomies (breaching the intercostal muscles and parietal pleura) in the 5th intercostal space in the midaxillary line-the same technique and landmarks as for conventional chest drains.

- Note: The procedure is stopped at this point if tension pneumothorax is decompressed and cardiac output returns.

(4) Connect the thoracostomies with a deep skin incision following the 5th intercostal space (fig 2).

(5) Insert two fingers into a thoracostomy to hold the lung out of the way while cutting through all layers of the intercostal muscles and pleura towards the sternum using heavy scissors. Perform this on left and right sides leaving only a sternal bridge between the two anterolateral thoracotomies.

(6) Cut through the sternum or xiphoid using the heavy scissors. If unable to cut through bone with scissors, use the Gigli saw (serrated wire) as follows:

- Pass the large clamp/forceps under the sternum

- Grasp one end of the Gigli saw with the clamp/forceps and pull back under sternum

- Connect saw handles and with smooth, long strokes cut through the sternum from inside out

(7) Open the "clam shell" using one or two large self retaining retractors/rib spreaders from the full thoracotomy set (fig 3 ). If this is not available, the incision can be held open manually by one or two gloved assistants. The retractor should be opened to its full extent to provide adequate exposure of the chest cavity with access to all areas. If exposure is inadequate the incisions need to be extended posteriorly.

(8) Lift ("tent") the pericardium with clamp/ forceps and make a large midline longitudinal incision using scissors. This approach minimises the risk of damage to the phrenic nerves, which run in the lateral walls of the pericardial sac. Making the incision too short will prevent full access to the heart (see fig 3). intravenous access, etc should be performed by other members of the trauma team and not delay the thoracotomy. 


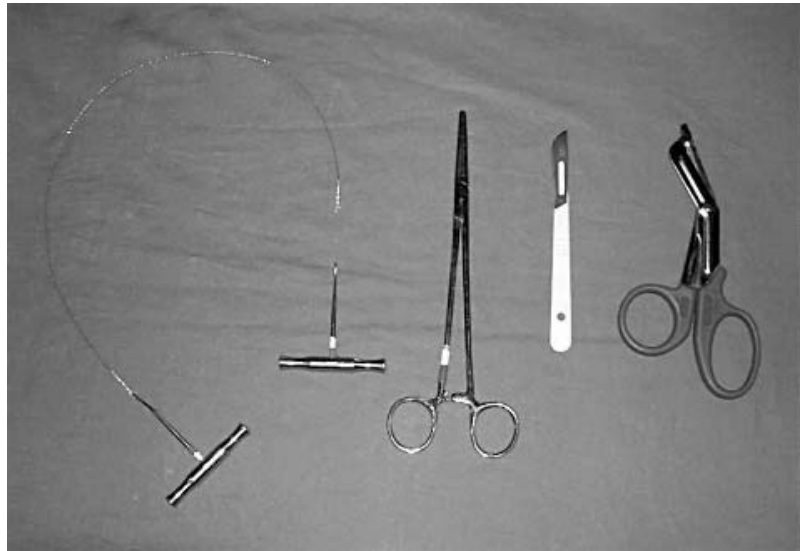

Figure 1 Equipment: Gigli saw, large clamp/forceps (or equivalent), large scalpel, and large scissors.

(9) Evacuate all blood and clot present, then inspect the heart rapidly but systematically for the site of bleeding.

(10) One of three scenarios are now likely:

(a) The heart will begin to beat spontaneously with a return of cardiac output. In this situation any cardiac wounds should be closed as described below.

(b) The heart begins to beat slowly with a considerably reduced cardiac output. In this situation wounds should be closed quickly, then attempt to improve cardiac output with supplementary internal cardiac massage and inotropic support.

(c) The heart remains in asystole. In this case wounds should be quickly closed and then attempts made to restart the heart as in step 10b. Simply flicking the heart may produce a return of contractions.

(11) When massage is required it must be of optimal quality. The authors' preference is a two handed technique. One flat hand is applied to the posterior surface of the heart and one on the anterior surface. Blood is "milked" from the apex upwards at a rate of 80 beats per minute. Alternatively a single hand can be used if it is large enough. Using this technique straight fingers are applied to the posterior surface of the heart, the apex is positioned in the palm of the hand and the thumb on the anterior surface. Whichever technique is used

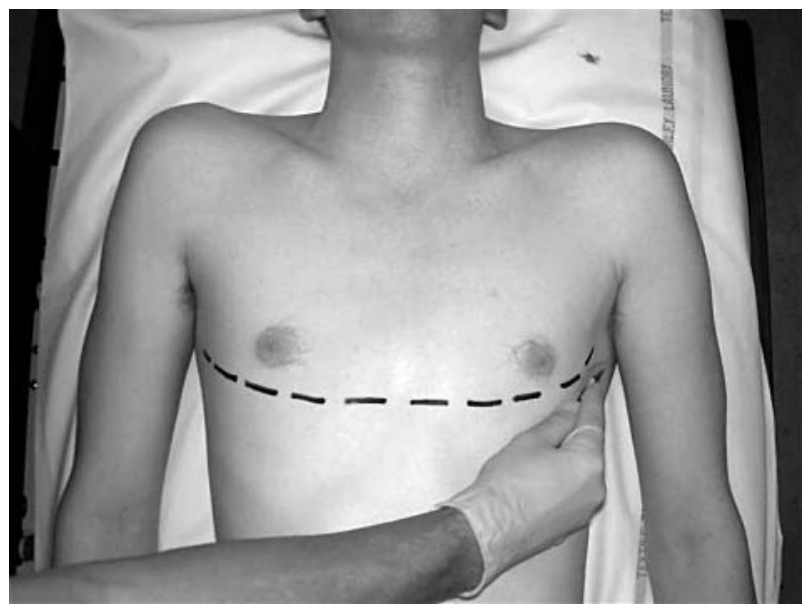

Figure 2 The clam shell incision. ensure that the heart remains horizontal during massage. Lifting the apex of the heart too far out of the chest can prevent venous filling. An assistant can compress the aorta against the spinal column using a thumb or fingers to maximise coronary and cerebral perfusion (fig 3).

(12) Control any bleeding:

- Holes less than $\mathrm{l} \mathrm{cm}$ can usually be occluded temporarily using a finger or gauze swab. If this is successful no other method should be attempted.

- For larger defects, a Foley urinary catheter can be passed through the hole then inflated and gently pulled back. This technique reduces the volume of the ventricular cavity (with subsequent reduction in stroke volume) therefore only a small volume $(<10 \mathrm{ml})$ should be used in the balloon. Ensure that the catheter is clamped to prevent blood loss from it. If a catheter is used in this way, a "giving set" can be attached to permit rapid volume infusion directly into the heart.

- If bleeding cannot be controlled with finger/gauze/Foley catheter, it may be necessary to close the defect with large sutures, but it should be emphasised this is a last resort as there is a risk of occluding coronary arteries. If sutures are used the minimum required to achieve haemostasis facilitated by finger/gauze/Foley catheter should be used. Non-absorbable size $0 / 0$ or $1 / 0$ monofilament or braided are appropriate; take $1-2 \mathrm{~cm}$ "bites".

(13) If defibrillation is required use internal paddles with an initial energy level of 10 joules. If these are not available, close the clam shell and defibrillate using conventional external pads.

(14) If the procedure is successful the patient may begin to wake up so be prepared to provide immediate anaesthesia.

(15) Restoration of circulation will be associated with bleeding, particularly from the internal mammary and intercostal vessels. Large bleeders may be controlled with artery forceps.

(16) Once perfusion has been restored the patient should be moved to theatre (optimally a cardiothoracic facility although this will depend on local expertise) for definitive repair.

\section{DISCUSSION}

The procedure and equipment used to perform a thoracotomy in a cardiothoracic operating theatre is highly specialised. To

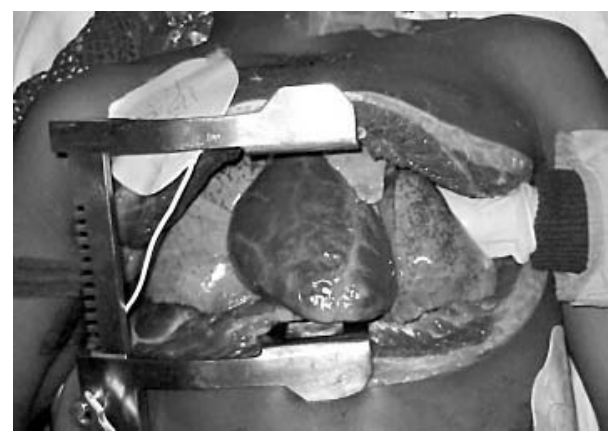

Figure 3 Open chest. Rib spreaders in situ. Central heart. In this picture the pericardium has been displaced by internal cardiac massage and lies behind the heart. Gloved hand compressing the aorta. 
a non-cardiothoracic surgeon this may give rise to anxiety and a reluctance to perform this intervention in an emergency setting. Patients with penetrating chest trauma resulting in cardiac arrest caused by tension pneumothorax or cardiac tamponade have potentially reversible pathology. Bilateral thorocostomies reliably rule out tension pneumothorax and should be performed even if needle decompression has been unsuccessful. This approach to tension pneumothorax has the additional advantage that the thorocostomies are also the start of the clam shell thoracotomy.

We have found needle periocardiocentesis to be ineffective because of the frequent presence of extensive clot in the pericardial sac. In patients without a cardiac output attempts at needle pericardiocentesis delay definitive intervention and therefore do not form part of our procedure.

A traditional left anterolateral thoracotomy has the advantage of quick and simple access to the pericardium, but has a number of disadvantages. Exposure of the heart is limited and will only permit visualisation of the apex and the anterior surface of the left ventricle, with a "side on" view. Once the tamponade has been relieved, identification of the site of bleeding may be difficult for the non-specialist. This may lead to continued and fatal haemorrhage. Making a large incision in the chest wall is the easy part of an emergency thoracotomy. The real challenge lies in correction of the underlying pathology. The left anterolateral approach is very effective for immediate relief of tamponade, but does not maximise the chances of a non-specialist being able to identify and correct the underlying problem.

Median sternotomy is the preferred approach by the cardiothoracic surgeon because it is rapid (under two minutes), can be performed on the supine patient, and gives complete access to the mediastinum and thoracic cavity. However, for the non-specialist it requires the use of complex, unfamiliar, and often unavailable equipment, so is not a practical option. Access to structures in the upper mediastinum is not relevant outside a cardiothoracic centre, as pathology in this area is rarely correctable by a nonspecialist surgeon.

The "clam shell" (bilateral anterior) thoracotomy described provides excellent exposure of the heart and lower mediastinum. Importantly it can be performed on the supine patient and also provides access to both pleural cavities. It allows the operator to view the anatomy from the front, making orientation easy. Using this technique a non-cardiothoracic surgeon should realistically be able to access the pericardium in two to three minutes. The procedure would normally be undertaken by middle grade or consultant medical staff. Theoretical understanding of the procedure can be obtained by reading this paper. Opportunity for practice is extremely limited. The first time the procedure is performed it is likely to be for real.

Simplification of the set of instruments required is an important part of our technique. Resuscitation room "thoracotomy packs" typically contain a large and bewildering array of equipment. Unfamiliar equipment can lead to confusion, ${ }^{4}$ so we use a small number of instruments, most of which are in daily use by emergency physicians (fig 1).

It is important to have a realistic expectation of what can be achieved by emergency thoracotomy. The procedure tackles a single pathology_cardiac tamponade with a controllable wound in the heart. If the underlying injury is any more complex than this a good outcome is unlikely outside a specialist surgical centre with a fully equipped cardiothoracic theatre and cardiopulmonary bypass facilities.

There is currently little evidence to support one technique of emergency thoracotomy over another. Our clinical experience has led to the development of the approach described in this paper, showing how an infrequently performed, complex medical procedure-bilateral anterior thoracotomy — can be simplified. The procedure described has the advantages of being an extension of a familiar procedure (chest drainage), using a minimum of familiar equipment, to give wide exposure of anatomy in a familiar orientation and using a clear procedural guide.

While attempting to remove the mystique and anxiety surrounding emergency thoracotomy we would emphasise the importance of avoiding inappropriate intervention. Surgery should not be undertaken on unsalvageable patients (blunt injury or more than 10 minutes since loss of cardiac output). However, when faced with no alternative, our experience suggests that the clam shell thoracotomy using the simplified technique described in this paper can be a lifesaving procedure.

\section{CONTRIBUTORS}

All authors contributed to the writing of the paper.

\section{Authors' affiliations}

D Wise, G Davies, Department Emergency Medicine and Pre-hospital Care, Royal London Hospital, London, UK

T Coats, Academic Department of Emergency Medicine, Leicester Royal Infirmary, Leicester, UK

D Lockey, Departments of Anaesthesia and Intensive Care, Frenchay Hospital, Frenchay, Bristol, UK

J Hyde, Sussex Cardiac Centre, Royal Sussex County Hospital, Brighton, UK

A Good, Department of Emergency Medicine, Arrowe Park Hospital, Liverpool, UK

Funding: none.

Conflicts of interest: none declared.

\section{REFERENCES}

1 Asensio JA, Berne JD, Demetriades D, et al. One hundred five penetrating cardiac injuries: a 2-year prospective evaluation. J Trauma 1998;44:1073-82.

2 Coats TJ, Keogh S, Clark H, et al. Prehospital resuscitative thoracotomy for cardiac arrest after penetrating trauma: rationale and case series. J Trauma 2001;50:670-3.

3 Asensio JA, Murray J, Demetriades D, et al. Penetrating cardiac injuries: a prospective study of variables affecting outcomes. J Am Coll Surg 1998; 186:24-34.

4 Scull MJ, Ferris LE, Tu JV, et al. Problems for clinical judgement: 3. Thinking clearly in an emergency. J Can Med Associat 2001;164:1170-5. 